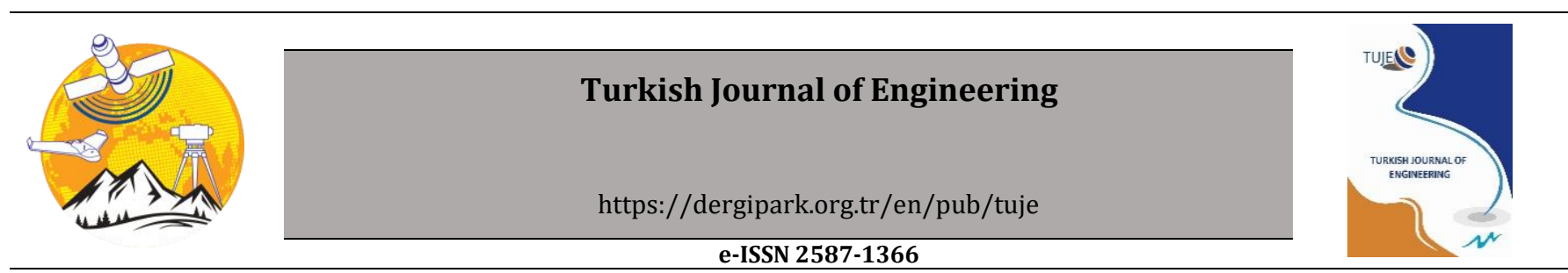

\title{
Image processing based autonomous landing zone detection for a multi-rotor drone in emergency situations
}

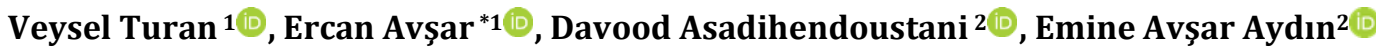 \\ ${ }^{1}$ Çukurova University, Faculty of Engineering, Department of Electrical and Electronics Engineering, Adana, Turkey \\ ${ }^{2}$ Adana Alparslan Türkeş Science and Technology University, Faculty of Aeronautics and Astronautics, Department of Aerospace \\ Engineering, Adana, Turkey
}

\section{Keywords}

Autonomus landing

Image processing

Object detection

UAV

\begin{abstract}
Flight safety and reliability improvement is an important research issue in aerial applications. Multi-rotor drones are vulnerable to motor failures leading to potentially unsafe operations or collisions. Therefore, researchers are working on autonomous landing systems to safely recover and land the faulty drone in on a desired landing area. In such a case, a suitable landing zone should be detected rapidly in for emergency landing. Majority of the works related with autonomous landing utilize a marker and GPS signals to detect landing site. In this work, we propose a landing system framework that involves only the processing of images taken from the onboard camera of the vehicle. First, the objects in the image are determined by filtering and edge detection algorithm, then the most suitable landing zone is searched. The area that is free from obstacles and closest to the center of the image is defined as the most immediate and suitable landing zone. The method has been tested on 25 images taken from different heights and its performance has been evaluated in terms runtime on a single board computer and detection precision and recall values. The average measured runtime is 2.4923 seconds and $100 \%$ of precision and recall values are achieved for the images taken from $1 \mathrm{~m}$ and $2 \mathrm{~m}$ The smallest precision and recall values are $79.1 \%$ and $81.2 \%$, respectively.
\end{abstract}

\section{INTRODUCTION}

The use of Unmanned Aerial Vehicles (UAV) has increased at an unpredictable rate in recent years. Although these devices have been used particularly in military applications for a long time, their use in nonmilitary applications such as fire extinguishing (Aydin et al. 2019), meteorological research (Martin et al. 2010; PropotoUAV 2019), exploration (Jakob et al. 2016; Heincke et al. 2019) and agricultural activities (Veroustraete 2015) have become very widespread nowadays. Quadrotors are the most common devices to use among UAV types due to their uncomplicated mechanical structures. Quadrotors can fulfill 3D motion tracking requirements with various technical systems such as Global Positioning System (GPS), ultrasonic detection, angular velocity sensors and linear accelerometers (Zhao and Wang 2012). Despite the use of these integrated systems and sensors, the control of the quadrotors is still one of the most difficult issues, but research and development activities continue in many research centers (Hoffmann et al. 2007; Zhao and Wang 2012). The vast majority of research activities address control issues during flying. The ultimate goal of UAV systems is to reach fully autonomous operations (Kim and Sukkarieh 2002).

One of the most important issue for UAV is autonomous landing in motion. Meanwhile, demands for automatic landing of drones on a defined position, safely, accurately, and without human's intervention, increase every day. It is always possible for a UAV to unavoidably face emergencies during flight, such as engine failure, interruption of data link from the ground and other unexpected accidents (strong wind, rain, etc.). Thus, forced landing measures should urgently be adopted in such situations. Methods of forced landing such as parachute and other flight termination systems can cause damage on the body of the multi-rotor (Fitzgerald et al., 2005). In addition, GPS signals are highly susceptible to be interrupted especially at lower altitudes (Lee et al. 
2012; Ho 2017), and in indoor environments. Furthermore, GPS signals are controlled by other nations, which causes vulnerability issues. For instance, in 29th October 2018, the GPS jamming that caused 46 drones to plummet during a display over Victoria Harbour caused at least HK\$1 million (US $\$ 127,500$ ) of damage, according to a senior official from the Hong Kong Tourism Board (Liteye 2018). As a result, some alternative methods were adopted to minimize the damage on that UAVs by enabling them to autonomously find a safe area suitable for landing. Some studies about forced landing in emergency situations for UAVs were presented in indoor environment without using GPS (Nemati et al. 2015).

In the past years, in design of emergency landing systems, effective algorithms of machine learning such as Support Vector Machines (SVM) and Artificial Neural Networks (ANN) were utilized in combination with digital image processing techniques for selection of appropriate landing site (Guo et al. 2014, Lunghi et al. 2015). It is observed that the above mentioned machine learning algorithms have different performance constraints, e.g. SVM is complex and requires huge computational power, whereas, ANN requires large training data set which corresponds to greater training time. Due to these constraints, both algorithms cannot meet the rapidly changing requirements for landing area selection in emergency flight conditions. For instance, in a previous study about detection of forced landing sites, 901 images were used to train and test of an ANN model (Fitzgerald and Walker 2005). Because the images fed to the ANN model were not representative of the training set, the final classification accuracy is very low when compared to training accuracy (Lu et al. 2013). Thus, that situation prevents it from being completely reliable.

Image processing is an appropriate and reliable way to find safe landing sites in case of an emergency. This is generally accomplished by detection of objects in the images taken from UAVs. Most of the related studies are about detection of some "marker" in an image (Barták et al. 2014; Cabrera-Ponce and Martinez-Carranza, 2017; Sani and Karimian 2017) which represents the desired landing spot. Utilization of a marker may not be a feasible way for emergency landing conditions. Since markers can be far from the UAV then it will take time to find the markers. For instance, the recent demonstration of package delivery using an UAV by Amazon shows the feasibility of an UAV sending a package to its consumer (AMAZON 2017). A marker which is placed by the consumer on the ground is used to allow the UAV to land safely. In some circumstances where the marker is unavailable or inappropriately placed, these vehicles need to be able to sense and avoid the surrounding objects in the environment and perform a smooth descent automatically. Without this capability, the safety of the surrounding animals, humans, and property cannot be ensured. Additionally, in some emergency situations, the UAV needs to land as quickly as possible. Therefore, it is essential to select a safe landing spot automatically and emergently, without depending on external systems.

Motivated by the above reasons, in this paper, an image processing method for object detection is proposed. The method is developed to work on the images taken from a drone's onboard camera. The aim is to rapidly detect a suitable landing zone in an unstructured and unknown environment. The method initially proposes a candidate landing zone at the center of the image. If the initial proposal is not suitable then new candidates in the neighboring area are evaluated until an available spot is detected (Fig. 1). The suitability of a spot is determined by existence of an object inside it. The object detection is accomplished by means of several image processing methods including edge detection, color processing, morphologic operations and thresholding. The major advantages of the method are (i) no requirement for a marker and (ii) no need for huge amount of data for training a model.

\section{METHOD}

\subsection{The Landing System Framework}

The use of multi-rotor drones has undeniably increased in the past decade, both in the military and civilian applications, thus raising a number of vital unsolved issues including safety and reliability. Engine malfunction or failures are among the common faults in multi-rotor drones, which apparently endanger the drone and the people's safety on the ground. In order to increase flight safety and reliability of drones, researchers are working on automation enhancement to safely recover the impaired drone (Lopez-Franco et al. 2017; Mazeh et al. 2018; Nguyen et al. 2019).

There are several challenges related with safe recovery or landing of impaired aerial vehicles. Majority of these challenges are about obstacle detection, suitable landing site detection/selection, fault detection and identification, characterizing the aircraft's new kinematic constraints, trajectory planning, and control of the faulty aircraft on the landing trajectory. To cover these challenges, an emergency landing system has been proposed according to Fig. 1. In fault or failure scenarios where continuation of flight is not possible or endangers the flight safety, the emergency flight system is triggered to recover drone's stability and safely land the drone on a suitable landing site.

The emergency landing system is translated to an architecture consisting of various subsystems that are capable of landing a faulty drone to a desired landing site along a designed trajectory without colliding to any human or animal. The architecture autonomously detects objects as well as possible landing sites, determines the most suitable landing site, develops the landing trajectory based on new kinematic and dynamic constraints of impaired drone, and controls it to the landing site, using onboard camera data and other common sensor information like IMU (Inertial Measurement Unit).

\subsection{Landing Zone Detection}

Emergency landing mode of the UAV is activated whenever a fault in any of the motors is detected. Then the onboard camera is immediately triggered to take a top-view photo of the ground in perpendicular direction. This image is the field of view (FOV) of the UAV and it constitutes the search space for finding a suitable landing 
zone. In such an emergency situation, locating the appropriate zone in a short time is very important. Therefore, the algorithm starts checking the suitability of center of the image which denotes the closest area to the UAV. If the image center is occupied by an object than it is labelled as "negative" and a neighborhood of the image center checked for suitability. The distance of the neighborhood to the image center is gradually increased until a vacant spot is found and that spot is labelled to be "positive". Next, the coordinates of this "positive" spot is sent to the flight controller to initialize the autonomous landing process. The steps for emergency landing are presented in Fig. 2.

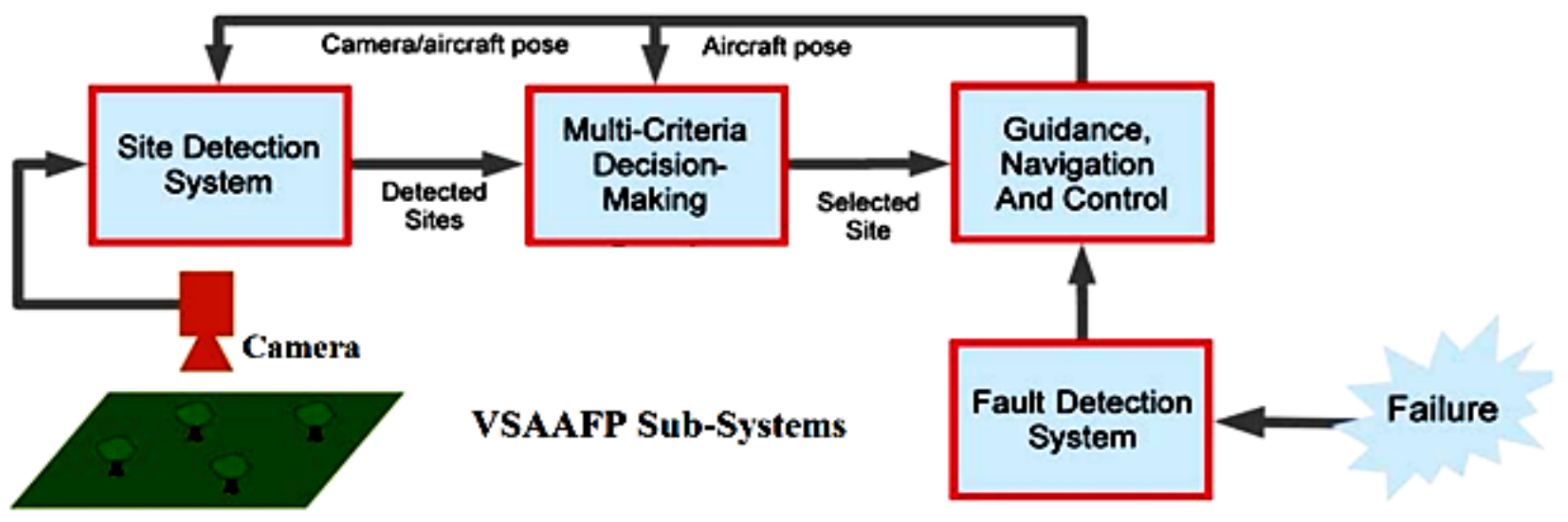

Figure 1. Emergency landing system architecture and subsystem

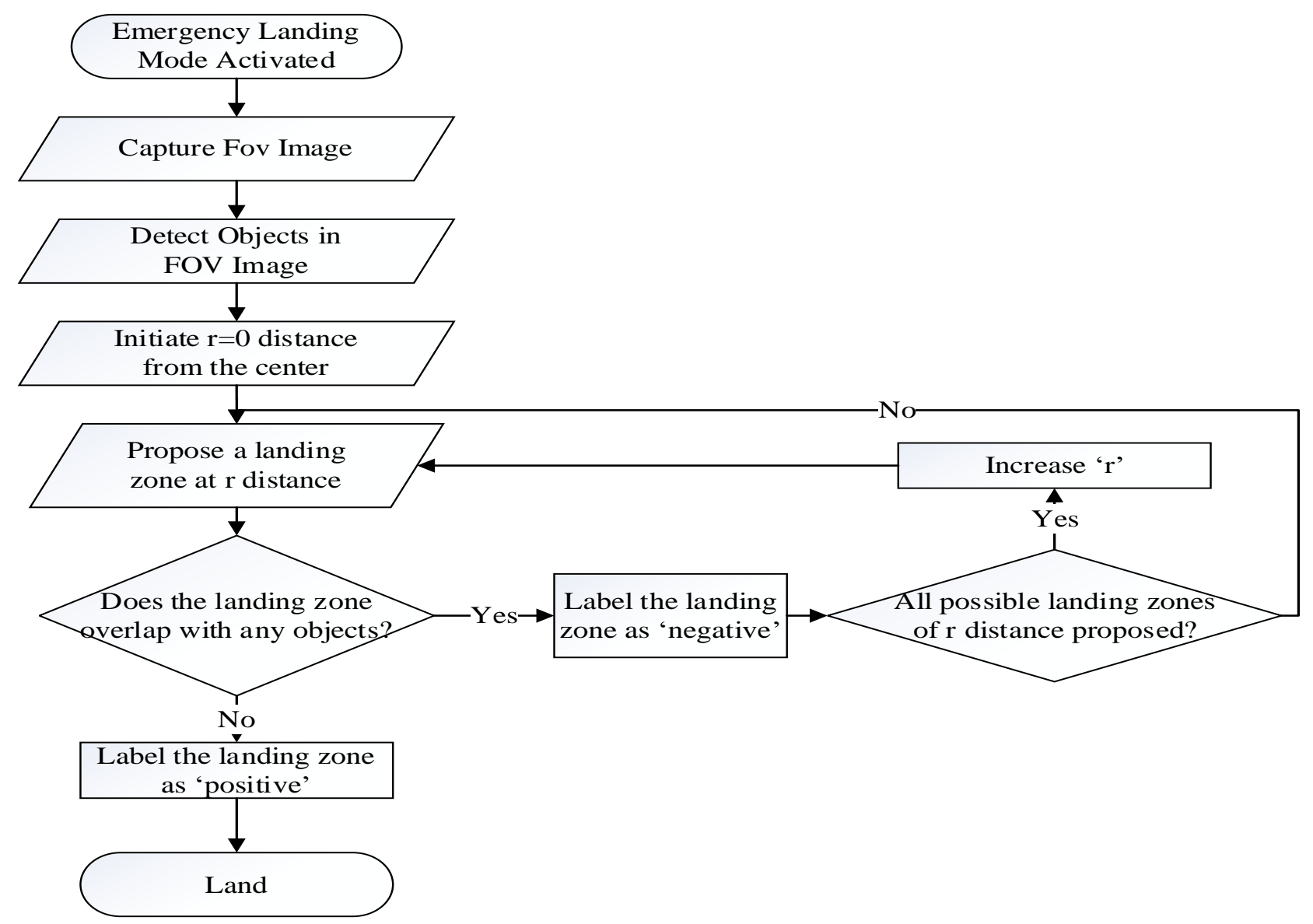

Figure 2. Suitable Landing site detection flowchart

\subsubsection{Object detection in FOV images}

The object detection starts with converting the FOV image into a grayscale image. This is necessary for preparing the image for canny operator, which is a method for edge detection to find objects on image. The
Canny edge detector is an edge detection operator that uses a multi-stage algorithm to detect a wide range of edges in images (Canny 1986). The advantage of using Canny edge detection technique over other well-known edge detection algorithms is that it gives better results even in noisy conditions (Kumar et al. 2015). 
The edge detection operator has four steps.

- Smooth an image with Gaussian filter.

- Calculate gradient magnitude and gradient direction.

- "Non - maximum suppression" to ensure the desired edge with one single pixel width.

- Determine two threshold values, and then select possible edge points and trace edges.

The first step in canny edge detector algorithm is to delete the noise in the frames by applying a Gaussian filter. In Canny algorithm, the Gaussian function is applied to smooth the image prior to edge detection. The filtering or smoothing operation actually supports two purposes. The first one is noise effect reduction prior to the detection of pixel intensity changes. The second purpose is setting the resolution or scale at which intensity changes are to be detected (Chen et al. 2014). These two purposes are necessary to improve the efficiency of edge detection method. In other words, Gaussian filtering helps reduction of detecting false edges.

Next step is to calculate the magnitude and gradient of the edges in the smoothed image. This is accomplished by filtering the smoothed image with a Sobel kernel in vertical and horizontal directions.

The complete scan of image is done after receiving gradient magnitude and direction, to remove any unwanted pixels, which may not establish the edge. In this step, just local maxima must be considered as edges through applying non-maximum suppression. Nonmaximum suppression exchanges the smoothed edges in the frame of the gradient magnitudes to sharp edges. This step is necessary to keep every local maximum in the gradient image, and remove any other detected edges which are possibly false detections.

The final step of canny edge detector algorithm is hysteresis thresholding. In this step, two threshold values are selected. The edges with intensity gradient greater than the maximum threshold are labeled as "sure-edge". Similarly, the edges with intensity gradient smaller than the minimum threshold are labeled as "nonedge". Other edges between these threshold values are labeled as "sure-edge" if they are connected to another "sure-edge", otherwise labeled as "non-edge". Obviously, this step removes small edges in the images that are possibly false detections. These threshold values are the only parameters of the method and are selected as 100 and 200 in this work.

Typically, the edge lines at the output of Canny method are thin lines. Therefore, dilation is applied to binary edge image as morphological operation to thicken the line of objects on image. Because thin line may cause errors when finding a landing zone. The binary image obtained after this operation is named as objects image. The outputs of these steps are given in Fig. 3.

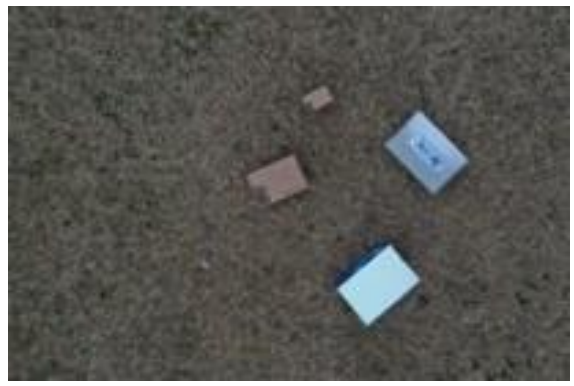

(a)

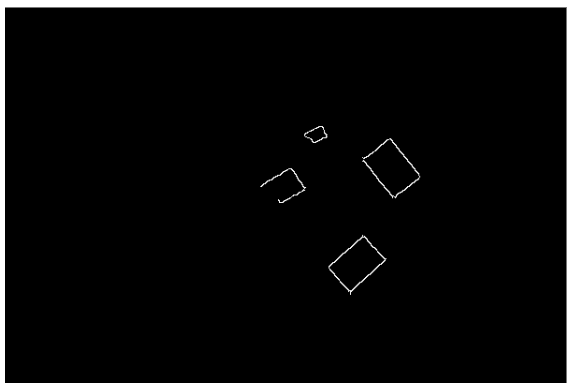

(b)

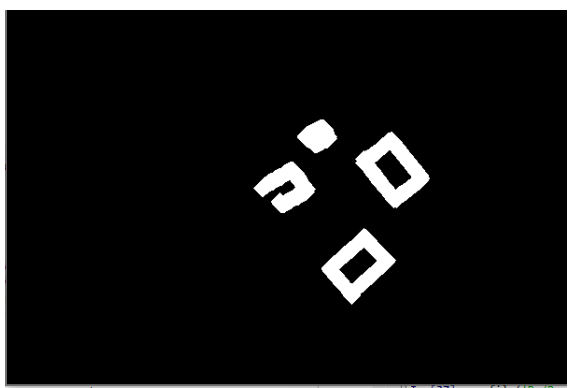

(c)

Figure 3. Steps of object detection. (a) FOV image, (b) Edge detection of FOV image, (c) Morphological operations are applied to binary image (objects image)

\subsubsection{Searching for a landing zone}

Once the objects in the FOV image are detected, the next step is to find the closest available zone for landing. The first parameter to consider for this step is the minimum dimensions of the suitable landing zone. Obviously, this parameter depends on the altitude at which the FOV image is taken. As illustrated in Fig. 4, in order to find a relation between the dimensions and the altitude, a calibration procedure is carried out on the images taken at five different altitudes. When the altitude is 1 meter, the ideal dimensions are 60x60 pixels; on the other hand, when the altitude is 5 meters, the ideal dimensions reduce to $20 \times 20$ pixels. The ideal dimensions for suitable landing zone change linearly with respect to altitude.

In a typical emergency landing scenario, a maximum altitude of 5 meters is appropriate for initialization of image processing tasks (Lee et al. 2014). Therefore, we used the same altitude value for our analysis as well. In case of triggering the emergency landing at a higher altitude, the UAV is initially brought to 5-meter altitude, then the image processing is activated.

After determining the optimal dimensions for landing, an available space for these dimensions is searched in the FOV image. The availability of a spot in the image is defined as the area in which no object is present. In order to check the availability, a binary mask is created. In the binary mask, an area with the optimal dimensions is made " 1 ", and all the remaining area is left as " 0 ". The area with " 1 " values in the binary image is the proposed region the availability of that region is checked. By observing the output of the logical AND operation between the binary mask and the objects image, it is possible to determine if a spot is available or not. If the logical AND operation returns " 1 " value, then it means that there is an overlap between any of the objects and the proposed region in the binary mask. Therefore, this proposed region is labeled as "negative". On the other hand, if a value of " 0 " is returned from the AND operation, then no overlap is present, hence the proposed region is labeled as "positive". 
When a proposed region is labeled as "negative", another region should be proposed immediately until a "positive" label is achieved. Since this work concerns only with the emergency situations, the location of the first proposed region is the middle of the FOV image which is the spot that is closest to UAV. If this spot is not available then a circular vicinity of the middle point is searched for availability, and the radius of the circle is gradually increased until an available spot is found. The steps of searching for a landing zone are given in Fig. 5.

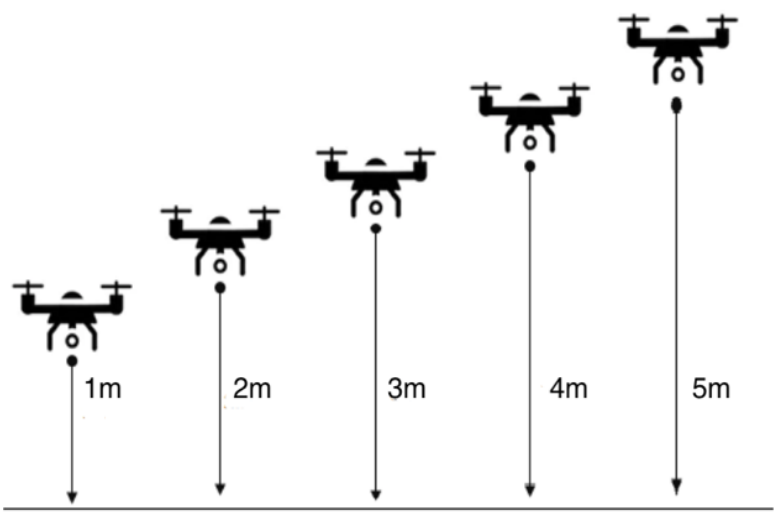

Figure 4. Ideal position to initialize the search of landing pad in different heights

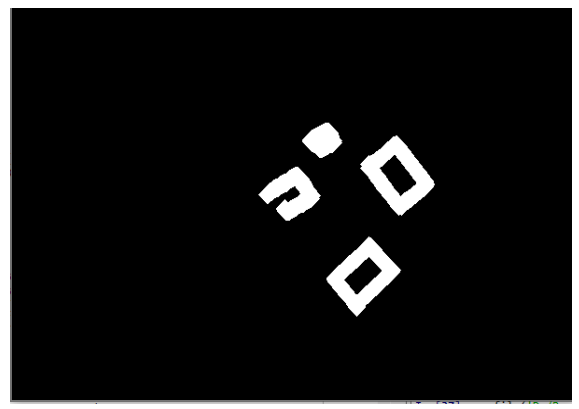

(a)

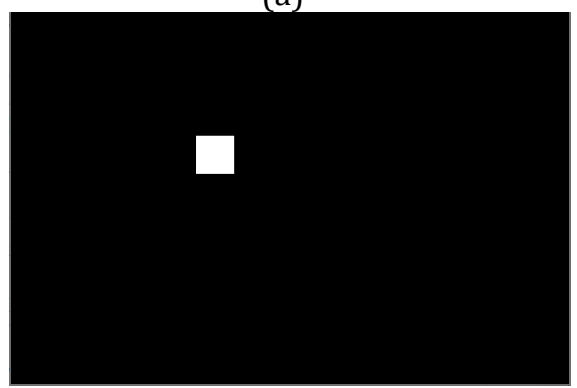

(d)

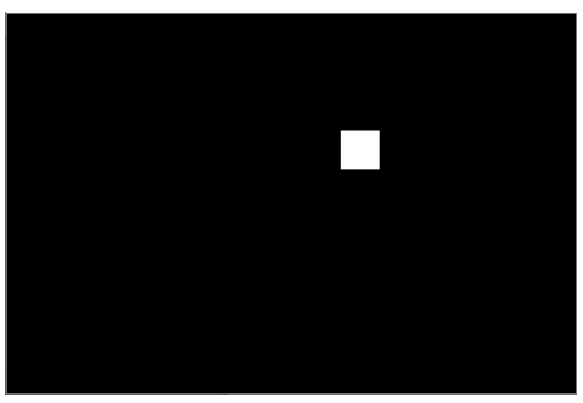

(b)

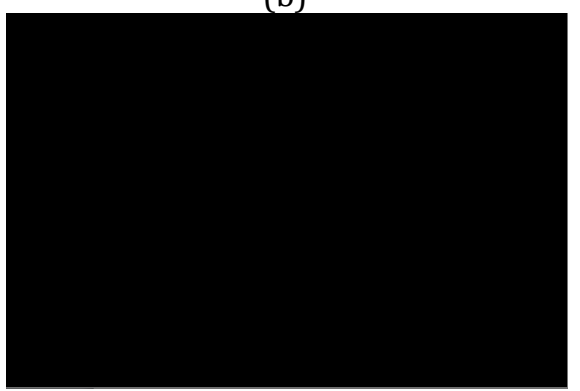

(e)

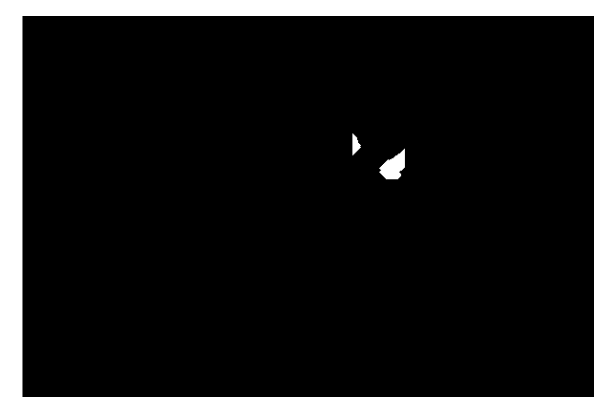

(c)

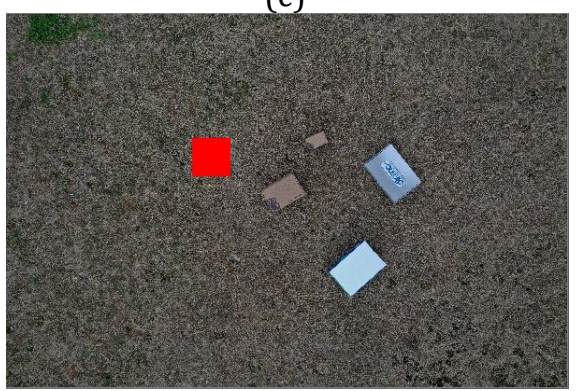

(f)

Figure 5. Steps of searching for a landing zone. (a) Objects image, (b) A binary mask for proposing a region, (c) Output of AND operation between $(a)$ and $(b)$ : "negative" labeling, (d) Another binary mask for proposing a region, $(\boldsymbol{e})$ Output of AND operation between $(a)$ and $(d)$ : "positive" labeling, $(\boldsymbol{f})$ Detected landing zone shown on the FOV image

\section{RESULTS AND DISCUSSION}

The method has been evaluated in terms of speed (i.e. runtime) and detection performance on a set of images taken from different altitudes. Here, we define the speed as the runtime of the algorithm to find a suitable landing zone in the image. On the other hand, the detection performance is defined as precision and recall values related with the object detection in the images.

As explained earlier, the landing zone detection is a part of autonomous landing system framework for emergency situations. Hence, the entire detection task has been experimented on a single board computer, which may easily be involved in a UAV system. The single board computer used in the experiments is Raspberry $\mathrm{Pi}$ 3 model B that has a Broadcom BCM2837B0 chipset, Cortex-A53 (ARMv8) 64-bit processor working at $1.4 \mathrm{GHz}$ frequency and $1 \mathrm{~GB}$ of memory. Besides, all the processed images have dimensions of $600 \times 400$ pixels.

During the experiments, it has been observed that the runtime of the algorithm for a particular image is different for consecutive runs. This may be because of the tasks related to operating system of the single board computer. Therefore, the algorithm has been run on all of the images for 10 separate times (i.e., 25 images $x 10$ times $=250$ observations) and then some statistical values are calculated on the observed runtimes. The range of the observations is [1.3940, 2.8478] seconds where the mean and standard deviation is calculated as 2.4923 seconds and 0.3899 seconds, respectively.

For calculating the precision and recall values, number of true detections (TP), false positive (FP) and false negative (FN) detections are determined. In order to determine these values, intersection over union (IoU) for all the detections are utilized. Calculation of IoU involves computing the overlap between the ground truth object and the detections. Since the purpose of this work is to find a suitable landing location, the threshold for IoU is set as 95\%. It means that if the IoU for a detection is smaller than this threshold then it is considered as FP, otherwise TP. On the other hand, any misdetections of an object are counted as FN. 
In the terminology of object detection, the precision is defined as the probability of the detected objects matching the actual objects. On the other hand, recall is a way to measure the probability actual objects being correctly detected.

Once the related quantities are determined, the precision and recall are calculated as follows:

$$
\text { precision }=\frac{T P}{T P+F P}
$$

$$
\text { recall }=\frac{T P}{T P+F N}
$$

There are five test images taken from five different altitudes, hence a total 25 images are used for evaluating the performance object detection. Besides, the algorithm is expected to work efficiently at different times of the day. Therefore, the same experiments were repeated by changing the brightness of the test images. The added brightness amounts vary from $-20 \%$ to $60 \%$. The related results are given in Table 1.

\begin{tabular}{|c|c|c|c|c|c|c|c|c|c|c|}
\hline \multirow{3}{*}{$\begin{array}{l}\text { Amount of } \\
\text { Brightness } \\
\text { Added (\%) }\end{array}$} & \multicolumn{10}{|c|}{ Altitude } \\
\hline & \multicolumn{2}{|c|}{$1 \mathrm{~m}$} & \multicolumn{2}{|c|}{$2 \mathrm{~m}$} & \multicolumn{2}{|c|}{$3 \mathrm{~m}$} & \multicolumn{2}{|c|}{$4 \mathrm{~m}$} & \multicolumn{2}{|c|}{$5 \mathrm{~m}$} \\
\hline & Precision & Recall & Precision & Recall & Precision & Recall & Precision & Recall & Precision & Recall \\
\hline-20 & 0.916 & 0.916 & 0.850 & 0.950 & 0.875 & 0.916 & 0.812 & 0.916 & 0.833 & 0.875 \\
\hline-10 & 0.916 & 1.000 & 0.900 & 1.000 & 0.875 & 0.916 & 0.875 & 0.937 & 0.875 & 0.916 \\
\hline 0 & 1.000 & 1.000 & 1.000 & 1.000 & 0.958 & 1.000 & 0.937 & 1.000 & 0.916 & 1.000 \\
\hline+10 & 1.000 & 1.000 & 1.000 & 1.000 & 0.958 & 1.000 & 0.937 & 1.000 & 0.916 & 1.000 \\
\hline+20 & 0.916 & 0.916 & 0.900 & 0.950 & 0.916 & 0.916 & 0.875 & 0.937 & 0.916 & 0.916 \\
\hline+40 & 0.875 & 0.916 & 0.850 & 0.900 & 0.875 & 0.875 & 0.812 & 0.875 & 0.833 & 0.875 \\
\hline+60 & 0.833 & 0.833 & 0.800 & 0.850 & 0.833 & 0.850 & 0.812 & 0.812 & 0.791 & 0.833 \\
\hline
\end{tabular}

Table 1. Precision and recall for different brightness levels and altitudes

As can be seen from the Table 1 that the highest precision and recall values are obtained at low altitudes ( $1 \mathrm{~m}$ and $2 \mathrm{~m}$ ) when no or $+10 \%$ brightness is added to the images. It is also notable that the results belonging to brightness addition of 0 and $+10 \%$ are identical for all of the altitudes. It means that the object detection is robust to addition of small amount of illumination. On the other hand, when the brightness or darkness of the images are increased, rate of FP and FN detections increase as well yielding decrements in precision and recall values. Preprocessing of the images using various filters or histogram equalization may be a useful step to improve the detection performance under different illumination levels.

When the table is analyzed according to different altitude levels, slight decrements in performance are observed as the altitude is increased. Thus, it may be a good practice to check the object locations during the emergency landing so that the detected locations may be updated when necessary. Furthermore, the recall value is generally higher than the precision value for all of the test instances. This means that the rate of FN detections is smaller than FP detections. When the application area of the framework is considered, burden of a FN detection is higher than a FP detection because misdetection of an object (i.e. FN) may cause a crash. However, on the other hand, detecting an object at an available area (i.e. FP) will just cause the runtime of the program to increase.

The overall accuracy of landing site detection is directly related with speed and performance of object detection step. As a result, high speed, precision, and recall values indicate the suitability of this method for autonomous landing.

\section{CONCLUSION}

An emergency landing system framework is proposed together with the details of the related image processing algorithm. The system is intended to work without any kind of markers showing the landing zone. Additionally, it does not utilize GPS signals, which may be unavailable under certain circumstances. It is based on direct detection of a suitable landing zone by processing of images taken from the onboard camera on the UAV. Since it does not involve any training and testing of a predictive model, the computational load is low and hence the corresponding response time is reasonable. On average, it takes around 2.5 seconds to make the detection for a single board computer and $100 \%$ of correct detection rate is achieved for the images taken from $1 \mathrm{~m}$ and $2 \mathrm{~m}$. The smallest precision and recall values are $79.1 \%$ and $81.2 \%$, respectively. These results show that the method is suitable for real-world scenarios. In addition, higher detection performance at lower altitudes means that the algorithm should be fast enough to make a final decision at 2 meters. In the future, the object detection may be run at different altitudes of the emergency landing process and update the landing trajectory accordingly. Also, the latencies in the data transfer pipeline may be considered for more accurate response time. This method is obviously intended to work on terrestrial zones. In other words, it may not detect water area, which is not a suitable zone for landing. Therefore, the algorithm may be improved to work on the images involving water area.

\section{ACKNOWLEDGEMENT}

This research was supported by Scientific Research Project Unit of Adana Alparslan Türkeş Science and Technology University with the project number of 19119001.

\section{REFERENCES}

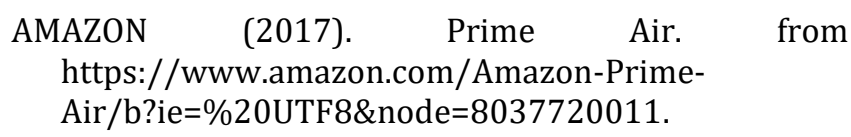


Aydin B, Selvi E, Tao J \& Starek M (2019). Use of FireExtinguishing Balls for a Conceptual System of Drone-Assisted Wildfire Fighting. Drones, 3(1). https://doi.org/10.3390/drones3010017

Barták R, Hraško A \& Obdržálek D (2014). On autonomous landing of AR.Drone: Hands-on experience. Proceedings of the 27th International Florida Artificial Intelligence Research Society Conference, FLAIRS 2014: 400-405.

Cabrera-Ponce A A \& Martinez-Carranza J (2017). A vision-based approach for autonomous landing. 2017 Workshop on Research, Education and Development of Unmanned Aerial Systems (REDUAS). Linköping, Sweden. DOI: 10.1109/REDUAS.2017.8101655

Canny J (1986). A Computational Approach to Edge Detection. IEEE Transactions on Pattern Analysis and Machine Intelligence PAMI-8(6): 679-698. DOI: $10.1109 /$ TPAMI.1986.4767851

Chen W, Yue H, Wang J \& Wu X (2014). An improved edge detection algorithm for depth map inpainting. Optics and Lasers in Engineering, 55: 69-77. https://doi.org/10.1016/j.optlaseng.2013.10.025

Fitzgerald D \& Walker R (2005). Classification of Candidate Landing Sites for UAV Forced Landings. AIAA Guidance, Navigation, and Control Conference and Exhibit, San Francisco, California.

Fitzgerald D, Walker R \& Campbell D (2005). A Vision Based Emergency Forced Landing System for an Autonomous UAV. Australian International Aerospace Congress, Melbourne, Australia.

Guo X, Denman S, Fookes C, Mejias L \& Sridharan S (2014). Automatic UAV Forced Landing Site Detection Using Machine Learning. 2014 International Conference on Digital Image Computing: Techniques and Applications (DICTA). Wollongong, NSW, Australia. DOI: 10.1109/DICTA.2014.7008097

Heincke B, Jackisch R, Saartenoja A, Salmirinne H, Rapp S, Zimmermann R, Pirttijärvi $M$ et al. (2019). Developing multi-sensor drones for geological mapping and mineral exploration: setup and first results from the MULSEDRO project. Geological Survey of Denmark and Greenland Bulletin 43. https://doi.org/10.34194/GEUSB-201943-03-02

Ho H W (2017). Autonomous landing of Micro Air Vehicles through bio-inspired monocular vision. PHD Thesis, ISBN: 978-94-6186-818-3

Hoffmann G M, Huang H, Waslander S L \& Tomlin C (2007). Quadrotor Helicopter Flight Dynamics and Control: Theory and Experiment. AIAA Guidance, Navigation and Control Conference and Exhibit, South Carolina

Jakob S, Zimmermann R \& Gloaguen R (2016). Processing of drone-borne hyperspectral data for geological applications. 2016 8th Workshop on Hyperspectral Image and Signal Processing: Evolution in Remote Sensing (WHISPERS). Los Angeles, CA, USA. DOI: $10.1109 /$ WHISPERS.2016.8071689

Kim J \& Sukkarieh S (2002). Flight Test Results of GPS/INS Navigation Loop for an Autonomous Unmanned Aerial Vehicle (UAV). Proceedings of the 15th International Technical Meeting of the Satellite Division of The Institute of Navigation (ION GPS 2002). Portland, OR, 510 - 517

Kumar K, Li J \& Khan S (2015). Comparative Study on Various Edge Detection Techniques for 2-D Image. International Journal of Computer Applications 119(22), 6-10.

Lee D, Lim H, Kim H J \& Kim Y (2012). Adaptive ImageBased Visual Servoing for an Underactuated Quadrotor System. Journal of Guidance, Control, and Dynamics, 35(4), 1335-1353.

Lee M R, Su S, Yeah J E, Huang H \& Chen J (2014). Autonomous landing system for aerial mobile robot cooperation. 2014 Joint 7th International Conference on Soft Computing and Intelligent Systems (SCIS) and 15th International Symposium on Advanced Intelligent Systems (ISIS). Kitakyushu, Japan, DOI: 10.1109/SCIS-ISIS.2014.7044826

Liteye (2018). HK\$1 million in damage caused by GPS jamming that caused 46 drones to plummet during Hong Kong show. from https://liteye.com/hk1million-in-damage-caused-by-gps-jamming-thatcaused-46-drones-to-plummet-during-hong-kongshow/.

Lopez-Franco C, Gomez-Avila J, Alanis A, Arana-Daniel N \& Villaseñor C (2017). Visual Servoing for an Autonomous Hexarotor Using a Neural Network Based PID Controller. Sensors, 17(8), 1865. https://doi.org/10.3390/s17081865

Lu A, Ding W \& Li H (2013). Multi-information Based Safe Area Step Selection Algorithm for UAV's Emergency Forced Landing. Journal of Software, 8(4), 995-1002.

Lunghi P, Ciarambino M \& Lavagna M (2015). VisionBased Hazard Detection With Artificial Neural Networks for Autonomous Planetary Landing. In 13th ESA/ESTEC Symposium on Advanced Space Technologies in Robotics and Automation, ASTRA

Martin S, Bange J \& Beyrich F (2010). Meteorological profiling of the lower troposphere using the research UAV "M²AV Carolo". Atmospheric Measurement Techniques Discussions 4, 705-716. DOI:10.5194/amt-4-705-2011

Mazeh H, Saied M, Shraim H and F. Clovis (2018). FaultTolerant Control of an Hexarotor Unmanned Aerial Vehicle Applying Outdoor Tests and Experiments. IFAC-PapersOnLine 51(22), 312-317. https://doi.org/10.1016/j.ifacol.2018.11.560

Nemati A, Sarim M, Hashemi M, Schnipke E et al. (2015). Autonomous Navigation of UAV through GPSDenied Indoor Environment with Obstacles. AIAA SciTech, Kissimmee, Florida

Nguyen N P, Mung N X \& Hong S K (2019). Actuator Fault Detection and Fault-Tolerant Control for Hexacopter. Sensors 19(21), 4721. https://doi.org/10.3390/s19214721

PropotoUAV (2019). Drones are being used for weather forecasting by meteorologists. from https://www.prophotouav.com/meteorologistsstorm-weather-drones/.

Sani M F \& Karimian G (2017). Automatic navigation and landing of an indoor AR. drone quadrotor using ArUco marker and inertial sensors. 2017 
International Conference on Computer and Drone Applications (IConDA), Kuching, Malaysia. DOI: $10.1109 /$ ICONDA.2017.8270408

Veroustraete F (2015). The Rise of the Drones in Agriculture. EC Agriculture 2(2), 325-327.
Zhao H \& Wang Z (2012). Motion Measurement Using Inertial Sensors, Ultrasonic Sensors, and Magnetometers With Extended Kalman Filter for Data Fusion. IEEE Sensors Journal - IEEE SENS J 12(5), 943-953. DOI: 10.1109/JSEN.2011.2166066

(C) Author(s) 2021.

This work is distributed under https://creativecommons.org/licenses/by-sa/4.0/ 